\title{
New exact soliton solutions, bifurcation and multistability behaviors of traveling waves for the $(3+1)$-dimensional modified Zakharov-Kuznetsov equation with higher order dispersion
}

\author{
Asit Saha ${ }^{1}$, Battal Gazi Karakoç², and Khalid K. Ali ${ }^{3}$ \\ ${ }^{1}$ Sikkim Manipal University \\ ${ }^{2}$ Nevsehir Haci Bektas Veli Universitesi \\ ${ }^{3}$ Al-Azhar University
}

October 27, 2021

\begin{abstract}
The goal of the present paper is to obtain and analyze new exact travelling wave solutions and bifurcation behavior of modified Zakharov-Kuznetsov (mZK) equation with higher order dispersion term. For this purpose, first and second simple methods are used to build soliton solutions of travelling wave solutions. Furthermore, bifurcation behavior of traveling waves including new type of quasiperiodic and multi-periodic traveling wave motions have been examined depending on the physical parameters. Multistability for the nonlinear mZK equation has been investigated depending on fixed values of physical parameters with various initial conditions. The suggested methods for the analytical solutions are powerful and benefical tools to obtain the exact travelling wave solutions of nonlinear evolution equations (NLEEs). Two and three-dimensional plots are also provided to illustrate the new solutions. Bifurcation and multistability behaviors of traveling wave solution of the nonlinear mZK equation with higher order dispersion will add some value in the literature of mathematical and plasma physics.
\end{abstract}

\section{Hosted file}

Modified Zakharov-Kuznetsov Eq..pdf available at https://authorea.com/users/443241/ articles/543263-new-exact-soliton-solutions-bifurcation-and-multistability-behaviorsof-traveling-waves-for-the-3-1-dimensional-modified-zakharov-kuznetsov-equation-withhigher-order-dispersion 